\title{
Explicit Calculation of Principle of Least Action
}

\author{
YUJi KAJIYAMA* \\ Gifu Shotoku Gakuen University, 1-1, Takakuwa-Nishi, Yanaizu, Gifu, 501-6194, Japan
}

Received: 22.03.2021 \& Accepted: 14.04.2021

Doi: $10.12693 /$ APhysPolA.139.704

*e-mail: kajiyama@gifu.shotoku.ac.jp

\begin{abstract}
In the standard way of analytical mechanics, the Euler-Lagrange equation as an equation of motion is derived from the principle of least action based on the variational principle. In this paper, we present an alternative way to describe the motion by explicitly solving an extreme value problem of the action without the variational principle. We assume that the position $x(t)$ is expressed by power series of time $t$ with an infinite number of unknown coefficients, and show that these coefficients can be correctly determined by the boundary conditions and the extremum conditions of the action by explicit calculations, which describe the actual motion. We will present the motion of a free particle, the motion under constant gravity, a harmonic oscillation, the time-dependent Lagrangian, and a non-linear force as examples.
\end{abstract}

topics: principle of least action, harmonic oscillator, non-linear force, analytical mechanics

\section{Introduction}

The principle of least action $[1,2]$ in analytical mechanics tells us that a particle moves along the path for which the action $S$ satisfies the stationary condition $\delta S=0$. The equation of motion, the Euler-Lagrange equation, is derived by solving the stationary conditions for the action, by applying the variational principle. In the context of physics education, simple and intuitive approaches to help students to understand the principle of least action have been studied by many [3-6]. Conditions of the minimality of the action are also discussed [7,8].

On the other hand, one can perform an explicit calculation of the action by assuming a specific form of function for $x(t)$. By solving the extremum condition for the action, one can obtain a function $x(t)$ which describes the realistic motion $[9,10]$. In [10], it is assumed that $x(t)$ has a form of $x(t)=A t^{n}$ (denoted by $y(t)$ in [10]) as a function of time $t$ with unknown coefficient $A$, to describe a motion under constant gravity. In this paper, we assume that $x(t)$ is written by an infinite power series of $t$, with an infinite number of unknown coefficients $a_{n}(n=0,1,2,3, \ldots)$, such as

$$
x(t)=\sum_{n=0}^{\infty} a_{n} t^{n} .
$$

Given the Lagrangian, one can explicitly calculate the action under this power series ansatz, and the action is a function of $a_{n}$. A different choice of the coefficients $a_{n}$ corresponds to a different path of the particle. We will show that the coefficients $a_{n}$ will be determined by solving two boundary conditions at $t=0$ and $t=T$ and the extremum conditions of the action, such that the corresponding function $x(t)$ correctly describes the motion. As examples, we discuss the one-dimensional motion such as a free particle, the motion under constant gravity, a harmonic oscillation, the time-dependent Lagrangian, and a non-linear force.

This paper is organized as follows. In Sect. 2, we show our formulation of the principle of least action in the context of coefficients $a_{n}$. In Sect. 3, we give five examples and show how the correct coefficients $a_{n}$ are determined. We conclude in Sect. 4 .

\section{Formulation}

In this section, we briefly follow the principle of least action and summarize our formulation for explicit calculation of the action, which will be applied in discussions below.

Here, we concentrate on the motion in one dimension along the $x$-axis. The Lagrangian $L(x(t), \dot{x}(t))$ depends on $x(t)$ and its time derivative $\dot{x}(t)$, and is defined as the difference of the kinetic energy and the potential energy $U(x(t))$, given by

$$
L(x(t), \dot{x}(t))=\frac{m}{2} \dot{x}(t)^{2}-U(x(t)),
$$

where $m$ is the mass of the moving object.

For the time region $0 \leq t \leq T$, the action $S$ is defined as

$$
S=\int_{0}^{T} \mathrm{~d} t L(x(t), \dot{x}(t)) .
$$


In order to find the correct path $x(t)$ which minimizes the action, we assume that $x$ is a function of time $t$ with independent coefficients $a_{n}(n=0$, $1,2, \ldots)$, that is,

$$
x=x\left(t, a_{0}, a_{1}, a_{2}, \ldots\right) .
$$

Since a different choice of $a_{n}$ corresponds to a different path of the particle, one has to find the set of $a_{n}$ which minimizes the action.

If one assumes that $x$ is a function of $N$ coefficients $a_{0}, a_{1}, a_{2}, \ldots a_{N-1}$, the action is a function of $T$ and $a_{n}, S\left(T, a_{0}, a_{1}, a_{2}, \ldots a_{N-1}\right)$. In a standard way for deriving the Euler-Lagrange equation, two boundary conditions for $x$ are imposed as $\delta x(0)=\delta x(T)=0$, which represents that the variations of $x$ vanish at the boundaries.

On the other hand, in our present approach, we will impose that two boundary conditions for $x$ at $t=0$ and $t=T$ are introduced by fixing the start point $x(0)$ and the endpoint $x(T)$ which reduce the number of independent coefficients by two. Therefore two coefficients, say $a_{0}$ and $a_{1}$, are eliminated from the action, and it is written as $S\left(T, a_{2}, a_{3}, \ldots a_{N-1}\right)$. The remaining $N-2$ coefficients will be determined by $N-2$ extremum conditions of the action,

$$
\frac{\partial S}{\partial a_{k}}=0, \quad k=2,3, \ldots, N-1 .
$$

In this way, $N$ conditions determine $N$ coefficients such that the boundary conditions and the extremum conditions are satisfied, without the variational principle and without solving the EulerLagrange equation.

In the next section, we will show with five examples that the coefficients $a_{n}$ obtained by this method correctly represent the motion, with $N \rightarrow \infty$.

\section{Examples}

Now we give five examples for explicit calculation of the principle of least action: (i) the motion of a free particle, (ii) the motion under constant gravity, (iii) a harmonic oscillation, (iv) the time-dependent Lagrangian, and (v) a non-linear force.

\subsection{Free particle}

When a particle of mass $m$ is moving along the $x$-axis without any potential, the Lagrangian is given by

$$
L=\frac{m}{2} \dot{x}(t)^{2} .
$$

For simplicity, we first assume that $x(t)$ is a quadratic function of time $t$ as

$$
\begin{aligned}
& x(t)=a_{0}+a_{1} t+a_{2} t^{2}, \\
& \dot{x}(t)=a_{1}+2 a_{2} t, \\
& \ddot{x}(t)=2 a_{2},
\end{aligned}
$$

where the independent coefficients $a_{0,1,2}$ will be determined by two boundary conditions of $x(t)$ and one extremum condition of the action.
If the particle is located at the origin $x=0$ at time $t=0$, and moves to $x=D$ at time $T$, then two boundary conditions eliminate two coefficients $a_{0}$ and $a_{1}$ as follows:

$$
\begin{aligned}
& x(0)=0 \Rightarrow a_{0}=0 \\
& x(T)=D \Rightarrow a_{1} T=D-a_{2} T^{2}
\end{aligned}
$$

which will be imposed in the action.

After a short calculation with the (8) substitution, one obtains the action $S$ as a function of $a_{2}$, i.e.,

$$
S=\int_{0}^{T} \mathrm{~d} t L=\frac{m}{6} T^{3} a_{2}^{2}+\frac{m}{2 T} D^{2} .
$$

Since the action $S$ is a quadratic function of $a_{2}$, it has the minimum if $T>0$. The extremum condition of the action

$$
\frac{\mathrm{d} S}{\mathrm{~d} a_{2}}=\frac{m}{3} T^{3} a_{2}=0
$$

gives $a_{2}=0$, and further also $a_{1}=D / T$ from (8). Applying the results to (7), finally we obtain

$$
\begin{aligned}
& x(t)=\frac{D}{T} t, \\
& \dot{x}(t)=\frac{D}{T}, \\
& \ddot{x}(t)=0,
\end{aligned}
$$

which describe the motion of a constant velocity $v=D / T$, as expected.

Next, for a general discussion, let us assume that $x(t)$ is a function of infinite power series of $t$ with an infinite number of coefficients $a_{n}$, given by

$$
\begin{aligned}
& x(t)=\sum_{n=0}^{\infty} a_{n} t^{n} \\
& \dot{x}(t)=\sum_{n=0}^{\infty} n a_{n} t^{n-1} \\
& \ddot{x}(t)=\sum_{n=0}^{\infty} n(n-1) a_{n} t^{n-2} .
\end{aligned}
$$

In this case, although all $a_{n}$ have to be determined by an infinite number of conditions, the method to be taken is the same as before. From two boundary conditions, two degrees of freedom vanish and the coefficients $a_{0}$ and $a_{1}$ can be expressed as

$$
\begin{aligned}
& x(0)=0 \Rightarrow a_{0}=0 \\
& x(T)=D \Rightarrow a_{1} T=D-\sum_{n=2}^{\infty} a_{n} T^{n} .
\end{aligned}
$$

By imposing (13), the action is obtained as

$$
\begin{array}{r}
S=\frac{m}{2} \sum_{n, \ell=0}^{\infty} \frac{n \ell}{n+\ell-1} a_{n} a_{\ell} T^{n+\ell-1}= \\
\frac{m}{2}\left(\sum_{n, \ell=2}^{\infty} K_{n \ell} a_{n} a_{\ell} T^{n+\ell-1}+\frac{D^{2}}{T}\right),
\end{array}
$$


List of coefficients for the kinetic term $K_{n \ell}$ and the potential terms $A_{n}, A_{n \ell}, B_{n}, B_{n \ell}, B_{n \ell j}$ and $B_{n \ell j m}$.

\begin{tabular}{c|l}
\hline \hline$K_{n \ell}$ & $\frac{(n-1)(\ell-1)}{n+\ell-1}$ \\
\hline$A_{n}$ & $-\frac{1}{3}+\frac{1}{n+2}$ \\
$A_{n \ell}$ & $\frac{1}{3}-\left(\frac{1}{n+2}+\frac{1}{\ell+2}\right)+\frac{1}{n+\ell+1}$ \\
\hline$B_{n}$ & $-\frac{1}{5}+\frac{1}{n+4}$ \\
$B_{n \ell}$ & $\frac{1}{5}-\left(\frac{1}{n+4}+\frac{1}{\ell+4}\right)+\frac{1}{n+\ell+3}$ \\
$B_{n \ell j}$ & $-\frac{1}{5}+\left(\frac{1}{n+4}+\frac{1}{\ell+4}+\frac{1}{j+4}\right)-\left(\frac{1}{n+\ell+3}+\frac{1}{\ell+j+3}+\frac{1}{j+n+3}\right)+\frac{1}{n+\ell+j+2}$ \\
$B_{n \ell j m}$ & $\frac{1}{5}-\left(\frac{1}{n+4}+\frac{1}{\ell+4}+\frac{1}{j+4}+\frac{1}{m+4}\right)+\left(\frac{1}{n+\ell+3}+\frac{1}{n+j+3}+\frac{1}{n+m+3}+\frac{1}{\ell+j+3}+\frac{1}{\ell+m+3}+\frac{1}{j+m+3}\right)$ \\
& $\quad-\left(\frac{1}{n+\ell+j+2}+\frac{1}{n+\ell+m+2}+\frac{1}{n+j+m+2}+\frac{1}{\ell+j+m+2}\right)+\frac{1}{n+\ell+j+m+1}$
\end{tabular}

where

$$
K_{n \ell}=\frac{(n-1)(\ell-1)}{n+\ell-1}
$$

which can also be indicated in Table I. The extremum conditions for the action are given by

$$
\frac{\partial S}{\partial a_{k}}=m \sum_{n=2}^{\infty} K_{n k} a_{n} T^{n+k-1}=0,
$$

for $k \geq 2$, where the coefficients are independent from each other, and the derivative of the coefficient is expressed by the Kronecker delta $\delta_{\ell k}$, as $\partial a_{\ell} / \partial a_{k}=\delta_{\ell k}$. In order to satisfy the condition (16) for arbitrary $T$ and $k \geq 2$, all coefficients $a_{n}$ in the summation must vanish, i.e., $a_{n}=0$ (for $n \geq 2$ ). As a reminder, the only non-vanishing coefficient obtained from (13) that reproduces (11) is $a_{1}=D / T$.

In this way, one can find the correct form of $x(t)$ which describes the realistic motion by assuming the form (12) of function of $x(t)$ with the imposed boundary conditions and extremum conditions.

\subsection{Motion under constant gravity}

As the second example, we consider a vertical motion of an object with mass $m$ under constant gravity with the gravitational acceleration $g$. The Lagrangian is given by

$$
L=\frac{m}{2} \dot{x}(t)^{2}-m g x(t)
$$

As in the previous discussion regarding (12), we assume that $x(t)$ and its derivatives are written as an infinite power series of $t$.

By imposing the boundary conditions, two coefficients $a_{0}$ and $a_{1}$ are expressed by the other coefficients as

$$
\begin{aligned}
& x(0)=0 \Rightarrow a_{0}=0 \\
& x(T)=0 \Rightarrow a_{1} T=D-\sum_{n=2}^{\infty} a_{n} T^{n},
\end{aligned}
$$

which is the same as (13) with $D=0$.
Now, one might think that this result corresponds to the motion of an object that is vertically thrown upwards from $x=0$ at $t=0$, and it returns to the starting point at $t=T$. However, here we are not assuming that this is such a motion. In the following discussions, we will find what motion actually occurs under the boundary conditions (18) and the extremum conditions of the action.

Substituting (12) into (17), and integrating the Lagrangian in terms of $t$, we obtain

$$
\begin{aligned}
S & =\frac{m}{2} \sum_{n, \ell=0}^{\infty} \frac{n \ell}{n+\ell-1} a_{n} a_{\ell} T^{n+\ell-1} \\
& -m g \sum_{n=0}^{\infty} \frac{1}{n+1} a_{n} T^{n+1} .
\end{aligned}
$$

Since the kinetic term in (19) is the same as that of a free particle, the same expression appears for $D=0$ and by imposing the boundary conditions (18). In turn, the second term of (19) can be written as

$$
\begin{aligned}
& -m g\left(\frac{1}{2} a_{1} T^{2}+\sum_{n=2}^{\infty} \frac{1}{n+1} a_{n} T^{n+1}\right)= \\
& \frac{1}{2} m g \sum_{n=2}^{\infty} \frac{n-1}{n+1} a_{n} T^{n+1},
\end{aligned}
$$

by imposing (18). Therefore, the action is given as

$$
\begin{aligned}
S & =\frac{m}{2} \sum_{n, \ell=2}^{\infty} K_{n \ell} a_{n} a_{\ell} T^{n+\ell-1} \\
& +\frac{m g}{2} \sum_{n=2}^{\infty} \frac{n-1}{n+1} a_{n} T^{n+1} .
\end{aligned}
$$

Differentiating the action with respect to $a_{k}$, we obtain the extremum conditions

$$
\frac{\partial S}{\partial a_{k}}=m T^{k+1}\left(\sum_{n=2}^{\infty} K_{n k} a_{n} T^{n-2}+\frac{g}{2} \frac{k-1}{k+1}\right)=0,
$$

for arbitrary $k \geq 2$ and $T$. Dividing (22) by $\frac{m(k-1) T^{k+1}}{(k+1)}$, one gets 


$$
\sum_{n=2}^{\infty} \frac{(n-1)(k+1)}{n+k-1} a_{n} T^{n-2}=-\frac{g}{2} .
$$

Since the right hand side (r.h.s.) of (23) is constant, the $k$ dependence on the left hand side (l.h.s.) of (23) must vanish. This implies that the only relevant term on the l.h.s. is $a_{2} \neq 0$, because the factor $k+1$ is canceled out when $n=2$, and $a_{n}=0$ for $n \geq 3$.

From (23) and (19), we obtain

$$
a_{2}=-\frac{1}{2} g, \quad a_{1}=\frac{1}{2} g T,
$$

while from (12),

$$
\begin{aligned}
& x(t)=-\frac{1}{2} g t^{2}+\frac{1}{2} g T t, \\
& \dot{x}(t)=-g t+\frac{1}{2} g T, \\
& \ddot{x}(t)=-g,
\end{aligned}
$$

respectively. For $T>0$, these equations describe the motion of an object that is thrown upwards with the initial velocity $v_{0}=g T / 2$, as expected.

\subsection{Harmonic oscillation}

As the third example, we consider the motion of a harmonic oscillator with the Lagrangian

$$
L=\frac{m}{2} \dot{x}(t)^{2}-\frac{m}{2} \omega^{2} x(t)^{2} .
$$

Here, we assume that $x(t)$ is written by an infinite power series of $\omega t$. Namely,

$$
\begin{aligned}
& x(t)=\sum_{n=0}^{\infty} a_{n}(\omega t)^{n}, \\
& \dot{x}(t)=\sum_{n=0}^{\infty} n \omega a_{n}(\omega t)^{n-1}, \\
& \ddot{x}(t)=\sum_{n=0}^{\infty} n(n-1) \omega^{2} a_{n}(\omega t)^{n-2} .
\end{aligned}
$$

The boundary conditions reduce two degrees of freedom as usual,

$$
\begin{aligned}
& x(0)=0 \Rightarrow a_{0}=0 \\
& x(T)=0 \Rightarrow a_{1} \omega T=-\sum_{n=2}^{\infty} a_{n}(\omega T)^{n},
\end{aligned}
$$

which later will be imposed in the action.

Substituting (27) into (26), we obtain the action

$$
\begin{aligned}
S & =\frac{m \omega}{2} \sum_{n, \ell=0}^{\infty} a_{n} a_{\ell}(\omega T)^{n+\ell-1} \\
& \times\left(\frac{n \ell}{n+\ell-1}-\frac{(\omega T)^{2}}{n+\ell+1}\right)= \\
& \frac{m \omega}{2} \sum_{n, \ell=2}^{\infty} a_{n} a_{\ell}(\omega T)^{n+\ell-1}\left(K_{n \ell}-A_{n \ell}(\omega T)^{2}\right)
\end{aligned}
$$

where (28) has been imposed in the last equality to eliminate $a_{0}$ and $a_{1}$ from the action. The extremum conditions $\partial S / \partial a_{k}=0(k \geq 2)$ are of the form

$$
\sum_{n=2}^{\infty} a_{n}(\omega T)^{n+k-1}\left(K_{n k}-A_{n k}(\omega T)^{2}\right)=0 .
$$

In Table I, one finds the calculated $A_{n \ell}$, together with the other coefficients.

In order to find the conditions of $a_{n}$ which satisfy (30), we make the following replacement $n=n^{\prime}+2\left(n^{\prime} \geq 0\right)$ in the first term including $K_{n k}$. Then, one gets

$$
\sum_{n^{\prime}=0}^{\infty} a_{n^{\prime}+2}(\omega T)^{n^{\prime}+k+1} K_{n^{\prime}+2, k} .
$$

In the case of the second term in (30) including $A_{n k}$, the summation can be taken from $n=0$, since $A_{1 k}=0$ and $a_{0}=0$. Thus, the condition (30) can be expressed (rewriting $n^{\prime}$ as $n$ ) by

$$
\sum_{n=0}^{\infty}(\omega T)^{n}\left(K_{n+2, k} a_{n+2}-A_{n k} a_{n}\right)=0,
$$

except for the factor $(\omega T)^{k+1}$. If one requires the second parentheses $(.$.$) to be zero for each n$, the trivial solution $a_{n}=0$ is obtained. In order to find the nontrivial solution for $a_{n}$, we assume the recurrence formula for $a_{n}$. Namely,

$$
a_{n+2}=f(n) a_{n} \text {. }
$$

and then (32) leads to

$$
\sum_{n=0}^{\infty} a_{n}(\omega T)^{n} g(n, k)=0,
$$

where

$$
g(n, k)=K_{n+2, k} f(n)-A_{n k},
$$

for arbitrary $T$ and $k \geq 2$. Since what we can only impose here is the boundary conditions (28) which are equivalent to $\sum_{n=0}^{\infty} a_{n}(\omega T)^{n}=0$, we find that the condition $(34)$ is satisfied if $g(n, k)$ is independent of $n$.

In order to find the conditions under which $g(n, k)$ is independent of $n$, we require $\partial g / \partial n=0$. As a result

$$
\begin{aligned}
& f(n)+(n+1) f^{\prime}(n)-\frac{1}{(n+2)^{2}}= \\
& \quad \frac{(n+1)(n+2) f(n)+1}{(n+k+1)(n+2)},
\end{aligned}
$$

where the r.h.s. contains $k$, while the l.h.s. does not. This implies that the $k$ dependence on the r.h.s. must vanish. Hence,

$$
f(n)=-\frac{1}{(n+1)(n+2)} .
$$

The recurrence formula $(33),(37)$, and $a_{0}=0$ followed by (28), give the relations

$$
a_{2 j}=0, \quad a_{2 j+1}=\frac{(-1)^{j}}{(2 j+1) !} a_{1},
$$

valid for $j=0,1,2, \ldots$ With the support of the first equation in (28), we obtain the final result

$$
x(t)=a_{1} \sum_{j=0}^{\infty} \frac{(-1)^{j}}{(2 j+1) !}(\omega t)^{2 j+1}=a_{1} \sin (\omega t),
$$


which describes motion of a simple harmonic oscillator with the angular velocity $\omega$, as expected. Since the remaining coefficient $a_{1}$ is the overall factor of the action, it cannot be determined by the extremum condition of the action.

\subsection{Time-dependent Lagrangian}

For a different class of the Lagrangian, let us consider the case of the time-dependent Lagrangian. If a resistance force is proportional to the velocity $f=-\alpha \dot{x}$ ( $\alpha$ is a constant), such as air resistance, the Lagrangian depends explicitly on time as

$$
L=\frac{m}{2} \dot{x}(t)^{2} \mathrm{e}^{\alpha t},
$$

and the equation of motion $m \ddot{x}=-\alpha \dot{x}$ is derived from the Euler-Lagrange equation. Solving it under the initial conditions

$$
\begin{aligned}
& x(0)=0, \\
& \dot{x}(0)=v_{0},
\end{aligned}
$$

one obtains

$$
x(t)=\frac{v_{0}}{\alpha}\left(1-\mathrm{e}^{-\alpha t}\right) .
$$

In several steps, we will now derive (42) based on the current approach. Firstly, we assume the replacement of $\omega \rightarrow \alpha$ in the position $x(t)$ and its derivatives given by $(27)$. Secondly, the factor $\mathrm{e}^{\alpha t}$ in (40) can be expanded as

$$
\mathrm{e}^{\alpha t}=\sum_{n=0}^{\infty} \frac{1}{n !}(\alpha t)^{n}
$$

Under the boundary conditions

$$
\begin{aligned}
& x(0)=0 \Rightarrow a_{0}=0 \\
& x(T)=D \Rightarrow a_{1} \alpha T=D-\sum_{n=2}^{\infty} a_{n}(\alpha T)^{n},
\end{aligned}
$$

one can perform the time integration of the Lagrangian, and obtain

$$
\begin{aligned}
& S=\frac{m}{2} \frac{\alpha}{\alpha T} \sum_{n=0}^{\infty} \frac{(\alpha T)^{n}}{n !} \\
& \times\left[\frac{D^{2}}{n+1}+2 D \sum_{\ell=2}^{\infty} a_{\ell}(\alpha T)^{\ell}\left(\frac{\ell}{n+\ell}-\frac{1}{n+1}\right)\right. \\
&\left.+\sum_{\ell, j=2}^{\infty} a_{\ell} a_{j}(\alpha T)^{\ell+j}\left(\frac{1}{n+1}-\frac{2 \ell}{n+\ell}+\frac{\ell j}{n+\ell+j}\right)\right] .
\end{aligned}
$$

From the extremum condition (5), and by imposing (44), we obtain

$$
\begin{gathered}
\sum_{n=0}^{\infty} \frac{(\alpha T)^{n}}{n !} \sum_{\ell=1}^{\infty} \frac{k \ell}{n+\ell+k-1} a_{\ell}(\alpha T)^{\ell}= \\
\sum_{n=0}^{\infty} \frac{(\alpha T)^{n}}{n !} \sum_{\ell=1}^{\infty} \frac{\ell}{n+\ell} a_{\ell}(\alpha T)^{\ell} .
\end{gathered}
$$

Note, importantly, that the 1.h.s. contains $k$, while the r.h.s. does not. Although (46) can be identically satisfied if one requires $n+\ell=1$, it cannot determine $a_{\ell}$. We will now find $a_{\ell}$ from the condition that the l.h.s. of (46) is independent of $k$.
Since the summation in the l.h.s. of (46) is valid for $\ell^{\prime} \geq 0$, then introducing $\ell^{\prime}=\ell-1$ (and again denoting $\ell^{\prime}$ as $\ell$ ) one can obtain

$$
-\alpha T \sum_{n, \ell=0}^{\infty} \frac{(\alpha T)^{n}(-\alpha T)^{\ell}}{n ! \ell !} \frac{k}{n+\ell+k} C_{\ell+1},
$$

where

$$
C_{\ell+1}=(-1)^{\ell+1}(\ell+1) ! a_{\ell+1} .
$$

If $a_{\ell+1}$ can be written as

$$
a_{\ell+1}=C \frac{(-1)^{\ell+1}}{(\ell+1) !} \quad(\ell \geq 0)
$$

with a constant $C$, then $C_{\ell+1}=C$ becomes independent of $\ell$. In this case, the summation in (47) is 1 because the term of $n=\ell=0$ remains, and the other terms are canceled out with each other. Therefore, we obtain

$$
\text { l.h.s. }=-\alpha T C \text {. }
$$

The constant $C$ can be determined using (44), then

$$
C=-\frac{D}{1-\mathrm{e}^{-\alpha T}}
$$

At the final stage, the result is

$$
x(t)=\frac{D}{1-\mathrm{e}^{-\alpha T}}\left(1-\mathrm{e}^{-\alpha t}\right),
$$

which corresponds to $v_{0}=\alpha D /\left(1-\mathrm{e}^{-\alpha T}\right)$.

In this way, the problem can be solved similarly if the Lagrangian depends explicitly on time, as in the case of resistance force proportional to velocity.

\subsection{Non-linear force}

Now, we present an example of an equation of motion with a non-linear term. To confirm that our present approach works correctly, we will consider the Lagrangian

$$
L=\frac{m}{2} \dot{x}(t)^{2}+x(t)^{2}+\frac{1}{2} x(t)^{4},
$$

of which an analytical solution exists, without worrying about the physical backgrounds. The equation of motion is $\ddot{x}=2\left(x+x^{3}\right)$. Under the initial conditions

$$
x(0)=0, \quad \dot{x}(0)=1,
$$

one obtains the analytical solution

$$
x(t)=\tan (t) \simeq t+\frac{1}{3} t^{3}+\frac{2}{15} t^{5}+\frac{17}{315} t^{7}+\ldots
$$

In several steps, we will now derive (55) based on our current approach.

We assume (12) as the position $x(t)$ and its derivatives, and (13) as the boundary conditions. Under these assumptions, the kinetic term of the action is given by (14), and the potential terms $S_{2}$ from the $x^{2}$ term and $S_{4}$ from the $x^{4}$ term are given by

$$
\begin{aligned}
S_{2} & =\frac{1}{3} D^{2} T+2 D \sum_{n=2}^{\infty} A_{n} a_{n} T^{n+1} \\
& +\sum_{n, \ell=2}^{\infty} A_{n \ell} a_{n} a_{\ell} T^{n+\ell+1},
\end{aligned}
$$


and

$$
\begin{aligned}
S_{4} & =\frac{1}{2}\left[\frac{1}{5} D^{4} T+4 D^{3} \sum_{n=2}^{\infty} B_{n} a_{n} T^{n+1}+6 D^{2} \sum_{n, \ell=2}^{\infty} B_{n \ell} a_{n} a_{\ell} T^{n+\ell+1}+4 D \sum_{n, \ell, j=2}^{\infty} B_{n \ell j} a_{n} a_{\ell} a_{j} T^{n+\ell+j+1}\right. \\
& \left.+\sum_{n, \ell, j, m=2}^{\infty} B_{n \ell j m} a_{n} a_{\ell} a_{j} a_{m} T^{n+\ell+j+m+1}\right]
\end{aligned}
$$

respectively, where the potencial coefficients $A_{n}$, $A_{n \ell}$ and $B_{n}, B_{n \ell}, B_{n \ell j}$ are presented in Table I.

Here we impose the extremum conditions (5) and the boundary conditions (13) as usual. We are aiming to confirm that the analytical solution (55) can be derived. We put $a_{1}=1$ which corresponds to the initial condition (54). Then, except for the overall factor $T^{k+1}$, we obtain the condition

$$
\begin{aligned}
& \sum_{n=2}^{\infty} K_{n k} a_{n} T^{n-2}+2 T\left(\frac{1}{k+2}-\frac{1}{3}\right)+2 \sum_{n=2}^{\infty} a_{n} T^{n}\left(\frac{1}{n+k+1}-\frac{1}{n+2}\right)+2\left[T^{3}\left(\frac{1}{k+4}-\frac{1}{5}\right)\right. \\
& \quad+3 \sum_{n=2}^{\infty} a_{n} T^{n+2}\left(\frac{1}{n+k+3}-\frac{1}{n+4}\right)+3 \sum_{n, \ell=2}^{\infty} a_{n} a_{\ell} T^{n+\ell+1}\left(\frac{1}{n+\ell+k+2}-\frac{1}{n+\ell+3}\right) \\
& \left.\quad+\sum_{n, \ell, j=2}^{\infty} a_{n} a_{\ell} a_{j} T^{n+\ell+j}\left(-\frac{1}{n+\ell+j+2}+\frac{1}{n+\ell+j+k+1}\right)\right]=0
\end{aligned}
$$

If we require that the coefficients of each order of $T$ vanish, then all $a_{n}$ are constants independent of $k$, and we obtain

$$
\begin{aligned}
& T^{0}: a_{2}=0, \quad T^{1}: a_{3}=\frac{1}{3}, \\
& T^{2}: a_{4}=0, \quad T^{3}: a_{5}=\frac{2}{15}, \\
& T^{4}: a_{6}=0, \quad T^{5}: a_{7}=\frac{17}{315}, \ldots .
\end{aligned}
$$

Together with $a_{0}=0$ and $a_{1}=1$, we found that the analytical solution (55) is obtained.

\section{Conclusions}

In this paper, we have presented an alternative way to describe the motion by explicitly solving an extreme value problem of the action without the variational principle. Assuming the power series ansatz for $x(t)$ with an infinite number of coefficients $a_{n}$, the action is expressed as a function of $a_{n}$. The value of the action varies by $a_{n}$, and there exists the set of $a_{n}$ which gives the extremum value of the action, and we have shown that it correctly describes the actual motion.

Since a different boundary condition gives a different set of $a_{n}$, one can obtain a different solution by imposing another boundary condition. Also one can assume a different function of $t$ for $x(t)$, such as the Fourier series instead of $t^{n}$. In this paper, we have concentrated our discussion on the case where an analytical solution exists to show that our approach works properly. It would be possible to extend our discussion to other cases even if analytical solutions do not exist, in the same way.

The method discussed here will be an alternative way of understanding the principle of least action, based not on the variational principle. Generalizing this approach is left to future work.

\section{References}

[1] R.P. Feynman, R.B. Leighton, M. Sands, The Feynman Lectures on Physics Vol. 2, Addison-Wesley, USA 1964.

[2] E.F. Taylor, Am. J. Phys. 71, 423 (2003).

[3] J. Hanc, S. Tuleja, M. Hancova, Am. J. Phys. 71, 386 (2003).

[4] J. Hanc, E.F. Taylor, S. Tuleja, Am. J. Phys. 72, 510 (2004).

[5] J.L. Brun, Eur. J. Phys. 28, 487 (2007).

[6] L.P. McGinness, C.M. Savage, Am. J. Phys. 84, 704 (2016).

[7] C.G. Gray, E.F. Taylor, Am. J. Phys. 75, 434 (2007).

[8] M. Moriconi, Am. J. Phys. 85, 633 (2017).

[9] D.E. Neuenschwander, Emmy Noether's Wonderful Theorem, The John Hopkins U.P., Baltimore (MD) 2011.

[10] J. Spandaw, Am. J. Phys. 81, 144 (2013). 